\title{
IMPLEMENTACIÓN DE LA METODOLOGÍA AGILE DATA warehouse en el Banco de Crédito del Perú
}

\author{
Tania Marino Jiménez \\ tmarinoj@icloud.com \\ Universidad San Ignacio de Loyola, Lima, Perú
}

Hoy en día, las empresas necesitan tomar decisiones de forma más rápida y soportadas por la tecnología de la información. El presente proyecto, que se efectuó en el Banco de Crédito del Perú (BCP) se desplegó a partir del 2015; consistió en la evaluación e implementación de un enfoque metodológico ágil para el desarrollo de los proyectos del data warehouse, usando la metodología Scrum. El trabajo se inició con una consultoría para identificar qué mejoras y cambios se tendrían que realizar a través de un nuevo modelo de atención. Con las propuestas de mejora se ejecutó un piloto para comprobar la efectividad de la metodología ágil; los beneficios fueron los siguientes: reducción del time to market, reducción del tiempo de ejecución y cumplimiento de plazos en la etapa de desarrollo, disponibilidad para atención de más requerimientos, garantía de la calidad del entregable debido a la participación constante y activa del usuario, reducción del riesgo de error y de implementaciones sin valor al negocio, entre otras ventajas. Luego se desplegó el modelo por medio de la formalización del proceso en la organización y se conformó un comité de gobierno ágil —integrado por los principales gerentes de negociospara velar por el cumplimiento del modelo de atención. Como resultado de este cambio en la forma cómo se gestionaban los proyectos, se logró la optimización de los costos de los proyectos.

Palabras clave: Modelo data warehouse tradicional / agile data warehouse / Scrum / Sprint

\section{Implementation of an agile methodology for data warehouse at the BCP}

Nowadays, companies need to make faster decisions, with the support of information technology. The present project, which was applied in the BCP approximately two years ago, consisted in the evaluation and implementation of an agile methodological approach for the development of data warehouse projects, using the Scrum methodology. The work began with a consultancy to identify what improvements and changes would have to be made through a new model of customer service. With the improvement proposals, a pilot was executed to check the effectiveness of the Agile methodology. The benefits were the following: reduction of time to market, reduction of execution time and fulfillment of deadlines in the development stage, availability to attend more requirements, guarantee of the quality of the deliverable due to the constant and active participation of the user, reduction of the risk of error and implementations without value to the business, among other benefits. The model was displayed through the formalization of the process in the organization and an agile governance committee was formed - integrated by the main business managers - to ensure compliance with the model of customer service. As a result of this change, in the project management, the optimization of its costs was achieved.

Keywords: traditional data warehouse / agile data warehouse / Scrum / Sprint 


\section{Introducción}

En el BCP las necesidades de información en data warehouse se atendían bajo los flujos establecidos de atención de proyectos, modelo creado por McKinsey en el 2009. Este enfoque tradicional motivaba que el tiempo transcurrido desde la fecha en que el usuario realizaba un pedido a la Unidad de Sistemas hasta la entrega operacional time to market ${ }^{1}$ fuera muy largo, con un promedio de 83 días para requerimientos pequeños; por ello, no se podían tomar decisiones cuando los usuarios de negocios lo requerían. Esta situación generó que los usuarios de negocios, para conseguir sus requerimientos, realizaran actividades de forma independiente, en ambientes de desarrollo llamados sandbox, los cuales no poseen la estabilidad necesaria para contener procesos críticos de negocio. Estos sandbox representaban el $39 \%$ de los ambientes productivos del data warehouse.

Ante la situación descrita, se optó por la implementación de un enfoque metodológico ágil para el desarrollo de los proyectos del data warehouse, con el fin de obtener beneficios directos en los costos de los proyectos (tiempo, calidad, comunicación) en comparación con el enfoque tradicional.

Figura 1. Organigrama del proyecto ágil data warehouse en el $\mathrm{BCP}$

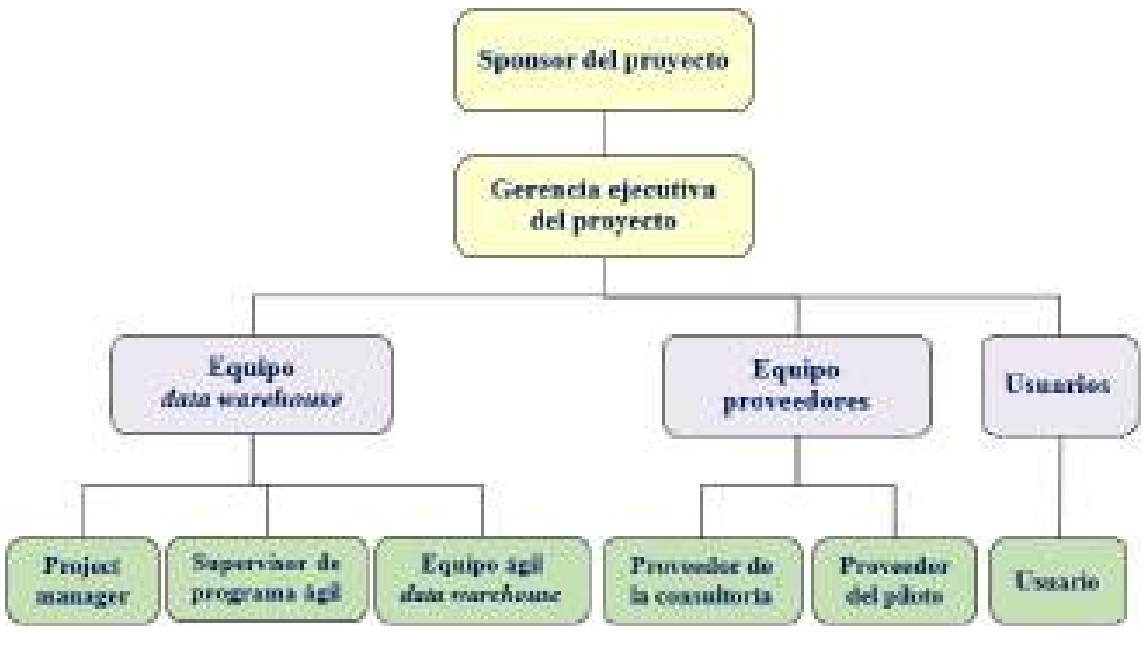

Elaboración propia

A continuación se muestra el roadmap general del proyecto, que fue plasmado en un cronograma en la etapa de planificación.

1 Tiempo en días que toma la atención de un pedido desde que el usuario lo solicita a la Unidad de Sistemas hasta que la solución es entregada. 
Figura 2. Roadmap del proyecto

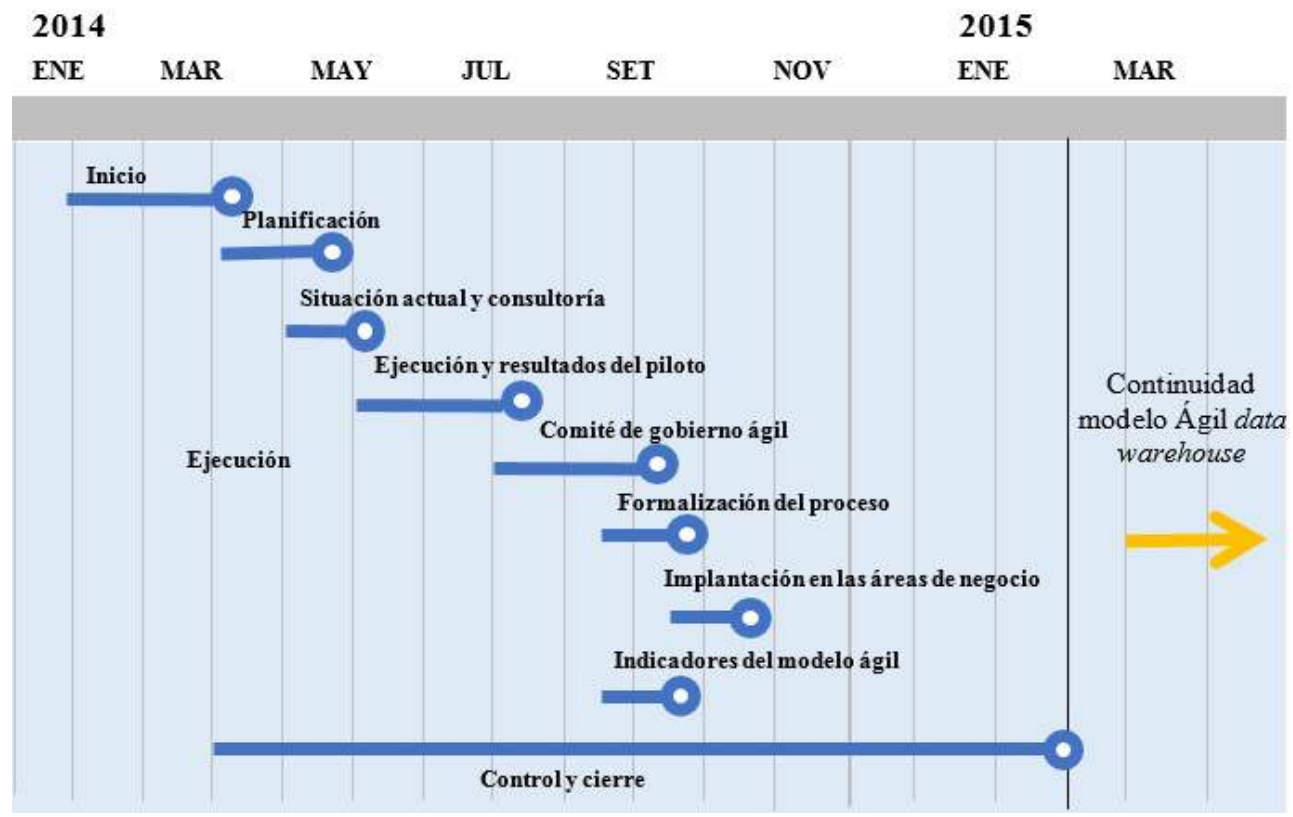

Fuente: BCP - Gerencia de Sistemas

\section{Conceptos preliminares}

\subsection{Método tradicional de desarrollo data warehouse}

El framework tradicional para ejecutar procesos del data warehouse es el modelo en cascada (waterfall). Mediante este enfoque se ordenan las etapas del proceso para el desarrollo de software, de tal forma que el inicio de cada etapa debe esperar a la finalización de la etapa anterior. Este método conlleva los siguientes pasos secuenciales:

i. Definición de requerimientos

ii. Modelamiento lógico de datos

iii. Mapeo de fuentes

iv. Modelamiento físico de datos

v. Diseño de integración de datos

vi. Testeo de integración de datos 
vii. Construcción capa de acceso

viii. Testeo capa de acceso

ix. Construcción business intelligence

$\mathrm{x}$. Testeo business intelligence

xi. Entrega

En la figura 3 se muestra el enfoque secuencial desde la definición del requerimiento hasta la entrega de la solución.

Figura 3. Modelo tradicional de desarrollo data warehouse - waterfall (cascada)

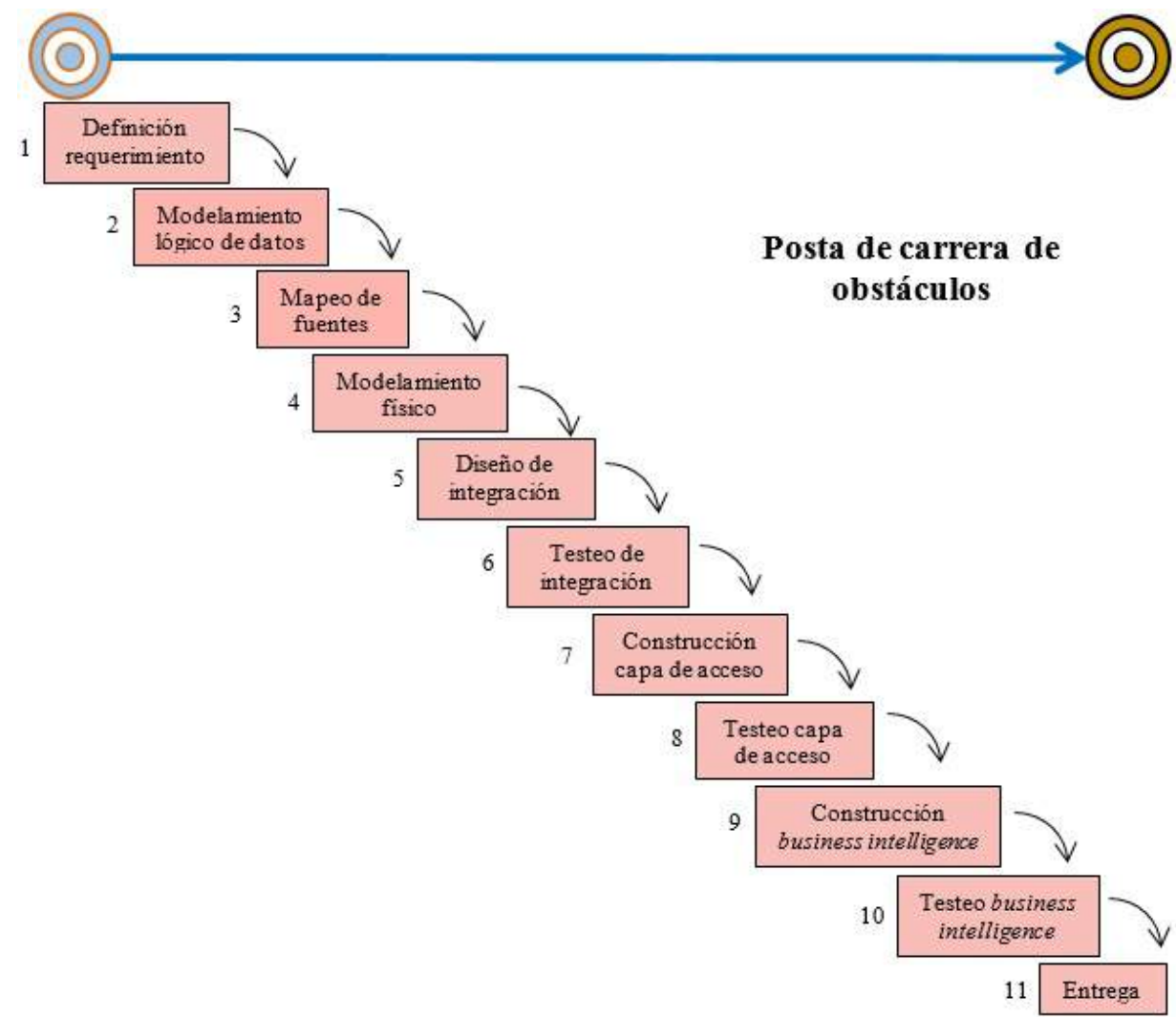

Fuente: Teradata (2014)

\subsection{Metodología Agile data warehouse}

Las metodologías ágiles son un conjunto de métodos basados en el desarrollo iterativo e incremental de software, donde los requerimientos y soluciones evolucionan con el 
tiempo según la necesidad del usuario. De esta manera, el trabajo es llevado a cabo mediante la colaboración de equipos autoorganizados y multidisciplinarios, gestionando entre ellos un proceso de toma de decisiones a corto plazo.

Las principales metodologías ágiles son Scrum, Programación Extrema (XP), Kanban entre otras, de las cuales Scrum es la más usada para modelos del data warehouse.

\section{Características:}
a) Equipos multifuncionales
b) Pequeñas iteraciones
c) Enfoque incremental
d) Prioridades del negocio y valor del cliente
e) Mejora continua

Figura 4. Ciclo de vida de proyectos ágiles data warehouse

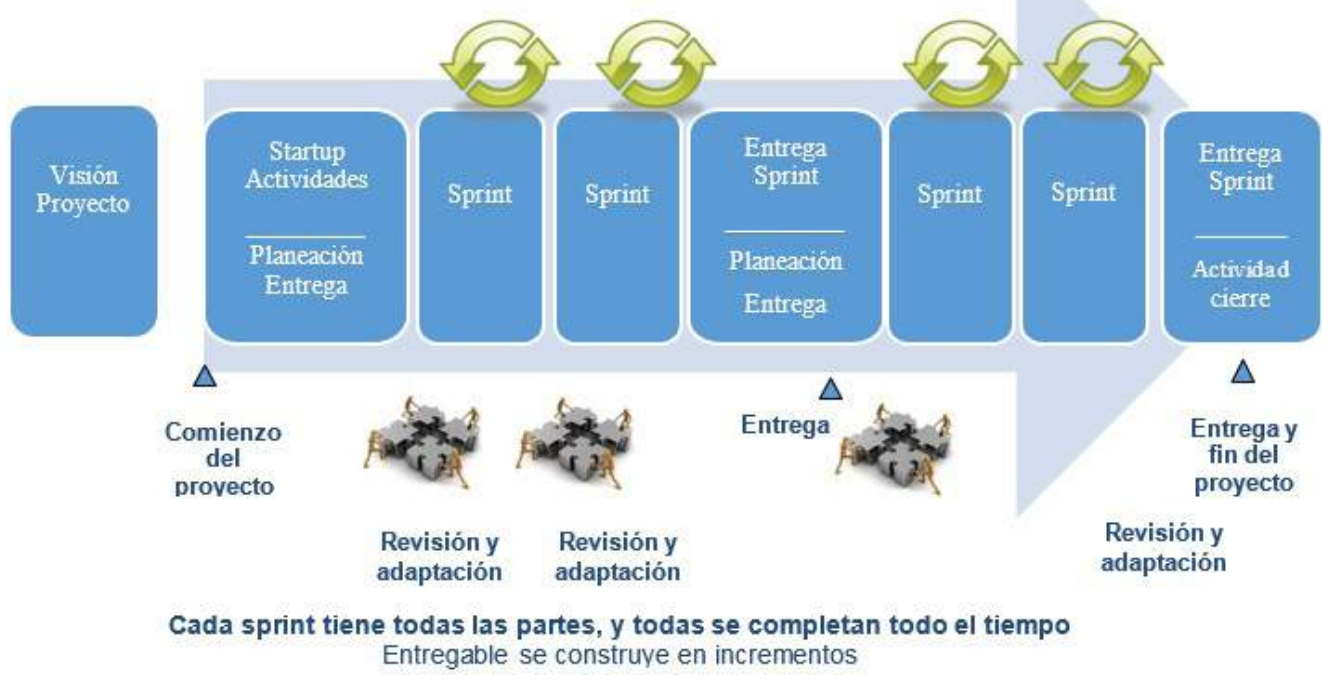


A continuación se detallan sus principios:

i. La prioridad más importante es la satisfacción del cliente a través de la entrega temprana y continua de un producto valioso.

ii. Los cambios son bienvenidos, aun cuando ocurran tarde durante el proceso.

iii. Entrega frecuente de resultados, entre dos semanas y dos meses, con preferencia hacia plazos más cortos.

iv. Las personas de las áreas de negocio y los desarrolladores deben interactuar en conjunto diariamente a lo largo del proyecto.

v. Los proyectos exitosos se arman con personas motivadas. Se les debe proveer del ambiente y soporte que necesitan, y confiar en que realicen el trabajo.

vi. El método más efectivo de compartir información dentro de un equipo es por medio de la conversación cara a cara.

vii. El avance se mide a través de la entrega de resultados.

viii. Un proceso ágil promueve el desarrollo sustentable. Los sponsors, desarrolladores y usuarios deben ser capaces de trabajar a este paso indefinidamente.

ix. La atención continua hacia la excelencia técnica y al buen diseño promueve la agilidad y rapidez.

x. La simplicidad es esencial, puesto que así se maximiza la cantidad de trabajo que no debe incluirse.

xi. La mejor definición de arquitectura, requerimientos y diseño viene de los propios equipos.

xii. El equipo debe analizar y proponer, cada cierto tiempo, cómo ser más efectivo y efectuar ajustes.

\subsection{SCRUM}

Scrum es un marco de desarrollo ágil, agile framework, centrado en proporcionar el mayor valor de negocio en el menor tiempo posible; actualmente es el enfoque más usado en la implementación de desarrollos ágiles. Tal como señalan Schwaber y Sutherland (2013) "es un enfoque que permite a las personas enfrentar y lidiar con problemas complejos en un entorno cambiante, siendo productivo y creativo a la vez, entregando un producto del más alto valor posible". Scrum se basa en un proceso de desarrollo incremental e 
iterativo, donde el negocio establece las prioridades, y los equipos se autoorganizan para determinar la mejor manera de cumplir con lo requerido. Entre las principales características se tiene:

i. Provee de muchas oportunidades para realizar evaluaciones y cambios durante el ciclo de vida del proyecto.

ii. Es un esfuerzo colaborativo que permite al usuario final estar informado e involucrado en el desarrollo.

iii. Provee de una entrega frecuente de valor al usuario.

iv. Permite compartir y revisar de forma rápida y repetida el producto de trabajo real.

Figura 5. El pizarrón Scrum

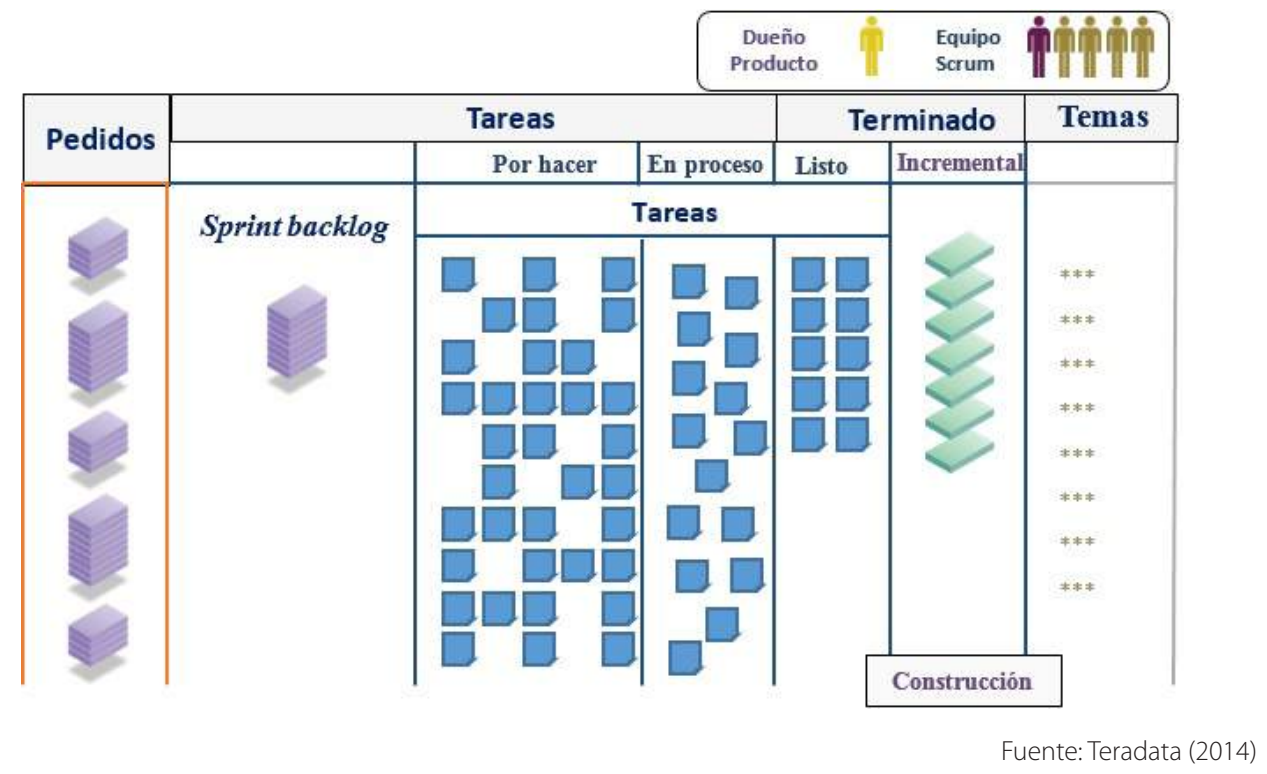

Sprint

Es una fase de implementación encuadrada en un espacio de tiempo (iterativo), cuya duración puede ser como mínimo un día y un máximo recomendado de un mes. Al término de cada sprint se dispondrá de un entregable usable, potencialmente listo para implementarse o para continuarse en la siguiente iteración. 


\section{Desarrollo del proyecto}

\subsection{Inicio}

Se efectuó la exploración de marcos de referencia de las metodologías ágiles y sus beneficios, lo cual permitió iniciar el proyecto con opciones viables de ejecución. Para esta etapa del proyecto se realizaron las siguientes actividades:

i. Se visitó el Data Warehousing Institute (TDWI) en Estados Unidos para conocer acerca de las metodologías ágiles. Gracias a ello, se obtuvo información de los principales métodos ágiles y se definió que Scrum era el indicado para un data warehouse.

ii. Se solicitó a un proveedor de Business Intelligence (BI) (Teradata) referencias sobre implementaciones de metodologías ágiles en Latinoamérica. Se detalla la información obtenida:

a) Bancolombia (Colombia), donde se implementaron métodos ágiles para el desarrollo de software.

b) Bimbo (México), donde se realizó la implementación de métodos ágiles para el data warehouse; además, un consultor de México, contratado por el $\mathrm{BCP}$, corroboró el éxito de los modelos ágiles.

iii. Se llevó a cabo una reunión con el $\mathrm{ClO}^{2}$, quien aprobó una consultoría para el relevamiento de la situación inicial de los requerimientos del data warehouse, y la correspondiente propuesta de mejora al proceso.

\subsection{Gestión del proyecto}

\subsubsection{Gestión del personal}

En la tabla 1 se muestra la información sobre el personal del proyecto y sus roles, las etapas en las cuales participó y la frecuencia de participación tanto en reuniones (como el caso del sponsor y la gerencia ejecutiva) como en el desarrollo del proyecto.

2 Chief Information Officer (CIO), responsable de la gestión estratégica de tecnologías de información. 


\section{Tabla 1. Recursos del proyecto}

\begin{tabular}{|c|c|c|}
\hline Recurso - rol & Etapas de participación & $\begin{array}{c}\text { Frecuencia } \\
\text { de participación }\end{array}$ \\
\hline Sponsor & Todo el proyecto & Mensual \\
\hline Gerencia ejecutiva del proyecto & Todo el proyecto & Semanal \\
\hline Project manager & Todo el proyecto & Diaria \\
\hline Supervisor de programa ágil & Todo el proyecto & Diaria \\
\hline $\begin{array}{l}\text { Analista } 1 \\
\text { Análisis y capa de control proveedor }\end{array}$ & Planificación, ejecución, seguimiento y cierre & Diaria \\
\hline $\begin{array}{l}\text { Analista } 2 \\
\text { Análisis y capa de control proveedor }\end{array}$ & Planificación, ejecución, seguimiento y cierre & Diaria \\
\hline $\begin{array}{l}\text { Analista } 3 \\
\text { Análisis y capa de control proveedor }\end{array}$ & Planificación, ejecución, seguimiento y cierre & Diaria \\
\hline $\begin{array}{l}\text { Analista } 4 \\
\text { Análisis y capa de control proveedor }\end{array}$ & Planificación, ejecución, seguimiento y cierre & Diaria \\
\hline Proveedor de la consultoría & Inicio y planificación & Diaria \\
\hline $\begin{array}{l}\text { Proveedor supervisor } \\
\text { Proveedor del piloto }\end{array}$ & Planificación, ejecución, seguimiento y cierre & Diaria \\
\hline $\begin{array}{l}\text { Proveedor analista } 1 \\
\text { Proveedor del piloto }\end{array}$ & Ejecución y cierre & Diaria \\
\hline $\begin{array}{l}\text { Proveedor analista } 2 \\
\text { Proveedor del piloto }\end{array}$ & Ejecución y cierre & Diaria \\
\hline $\begin{array}{l}\text { Proveedor analista } 3 \\
\text { Proveedor del piloto }\end{array}$ & Ejecución y cierre & Diaria \\
\hline $\begin{array}{l}\text { Proveedor analista } 4 \\
\text { Proveedor del piloto }\end{array}$ & Ejecución y cierre & Diaria \\
\hline $\begin{array}{l}\text { Proveedor analista } 5 \\
\text { Proveedor del piloto }\end{array}$ & Ejecución y cierre & Diaria \\
\hline $\begin{array}{l}\text { Proveedor analista } 6 \\
\text { Proveedor del piloto }\end{array}$ & Ejecución y cierre & Diaria \\
\hline
\end{tabular}




\subsubsection{Gestión de la comunicación}

Los comités que se implementaron para un seguimiento adecuado del proyecto, sus integrantes y la frecuencia de reuniones se muestran en la tabla 2.

Tabla 2. Plan de comunicación del proyecto

\begin{tabular}{|l|l|l|l|}
\hline \multicolumn{2}{|c|}{ Roles } & \multicolumn{2}{|c|}{ Reunión } \\
$\begin{array}{l}\text { Sponsor, gerencia } \\
\text { ejecutiva, project manager, } \\
\text { gerencia proveedor }\end{array}$ & $\begin{array}{l}\text { Comité ejecutivo } \\
\text { del proyecto }\end{array}$ & Mensual & $\begin{array}{l}\text { Se revisarán los avances en el nivel } \\
\text { estratégico, riesgos y problemas del } \\
\text { proyecto. Se podrán tomar decisiones en el } \\
\text { nivel de gerencia sobre el Proyecto }\end{array}$ \\
\hline $\begin{array}{l}\text { Gerencia ejecutiva, } \\
\text { project manager, gerencia } \\
\text { proveedor, supervisor de } \\
\text { programa ágil, proveedor } \\
\text { supervisor, proveedor } \\
\text { analista senior }\end{array}$ & Comité táctico & Quincenal & $\begin{array}{l}\text { Se revisarán los avances en el nivel táctico, } \\
\text { riesgos y problemas del proyecto. Se } \\
\text { buscarán acciones para mitigar los riesgos } \\
\text { y soluciones a los problemas. }\end{array}$ \\
\hline $\begin{array}{l}\text { Project manager, } \\
\text { supervisor de programa } \\
\text { ágil, proveedor supervisor, } \\
\text { proveedor analista senior, } \\
\text { analistas }\end{array}$ & $\begin{array}{l}\text { Comité } \\
\text { operativo }\end{array}$ & Semanal & $\begin{array}{l}\text { Se revisarán los avances en el nivel } \\
\text { operativo, se identificarán los riesgos y } \\
\text { problemas del proyecto. }\end{array}$ \\
\hline $\begin{array}{l}\text { Supervisor de programa } \\
\text { ágil, proveedor supervisor, } \\
\text { proveedor analista senior, } \\
\text { analistas }\end{array}$ & A solicitud & $\begin{array}{l}\text { Los equipos de trabajo podrán reunirse } \\
\text { cuando requieran revisar detalles técnicos. }\end{array}$ \\
\hline $\begin{array}{l}\text { Sponsor, gerencia ejecutiva } \\
\text { sistemas }\end{array}$ & $\begin{array}{l}\text { Trimestral } \\
\text { Comité de }\end{array}$ & $\begin{array}{l}\text { Se realizarán presentaciones al Comité de } \\
\text { Sistemas cuando este las requiera. }\end{array}$ \\
\hline
\end{tabular}

Elaboración propia

\subsubsection{Gestión de los factores críticos de éxito}

Se determinaron estos factores críticos de éxito del proyecto:

i. Gestión y seguimiento del proyecto con un control del cumplimiento de entregables.

ii. Disponibilidad de los usuarios de negocio participantes en la consultoría y el piloto.

iii. Trabajo conjunto entre el equipo de la unidad de Sistemas, los proveedores y los usuarios. 
iv. Revisión periódica del cronograma del proyecto, identificando riesgos y estableciendo planes de acción de manera conjunta.

v. Compromiso de la alta gerencia para el desarrollo exitoso del proyecto.

\subsubsection{Indicadores de seguimiento del modelo Agile data warehouse}

Se definieron los indicadores relevantes para llevar un control de la efectividad del nuevo proceso de atención de requerimientos, usando como criterio la perspectiva de los usuarios. Como no se contaba con un backlog de indicadores del nuevo modelo para poder definir las metas, se consideró que el objetivo sería mejorar el promedio de las metas del modelo base.

Tabla 3. Indicadores de seguimiento del modelo Agile data warehouse

\begin{tabular}{|c|c|c|c|c|}
\hline Nombre & Descripción & Fórmula & Meta & Responsable \\
\hline $\begin{array}{l}\text { Solicitudes } \\
\text { atendidas } \\
\text { por mes }\end{array}$ & $\begin{array}{l}\text { Porcentaje de atención } \\
\text { de solicitudes (total de } \\
\text { solicitudes menos el } \\
\text { backlog restante) con } \\
\text { respecto al total de } \\
\text { solicitudes. }\end{array}$ & $\frac{\text { Total solicitudes - Backlog }}{\text { Total solicitudes }}$ & $90 \%$ & $\begin{array}{l}\text { Gerencia } \\
\text { ejecutiva } \\
\text { del proyecto }\end{array}$ \\
\hline $\begin{array}{l}\text { Time to } \\
\text { market }\end{array}$ & $\begin{array}{l}\text { Mide el tiempo en días que } \\
\text { demora un pedido desde } \\
\text { que el usuario lo solicita } \\
\text { hasta que la solución es } \\
\text { entregada. }\end{array}$ & $\begin{array}{l}\text { Fechade - Fechade } \\
\text { entrega }\end{array}$ & < 83 días & $\begin{array}{l}\text { Project } \\
\text { manager }\end{array}$ \\
\hline $\begin{array}{l}\text { Desviación } \\
\text { de plazos }\end{array}$ & $\begin{array}{l}\text { Porcentaje de tiempo en } \\
\text { días que el cumplimiento } \\
\text { del plazo señalado para un } \\
\text { requerimiento se desvía de } \\
\text { su fecha de compromiso. }\end{array}$ & $\frac{\text { F. Entrega - F. Compromiso }}{\text { Tiempo de compromiso }}$ & $<10 \%$ & $\begin{array}{l}\text { Project } \\
\text { manager }\end{array}$ \\
\hline $\begin{array}{l}\text { Eficiencia } \\
\text { en la } \\
\text { ejecución }\end{array}$ & $\begin{array}{l}\text { Ratio del resultado del } \\
\text { time to market sobre el } \\
\text { esfuerzo en horas. }\end{array}$ & $\frac{\text { Time to market }}{\text { Esfuerzo }}$ & $<0,71$ & Usuarios \\
\hline
\end{tabular}

Elaboración propia

\subsection{Ejecución}

El proyecto se ejecutó con una estructura de fases, que se especifican a continuación:

\subsubsection{Relevamiento de la situación actual y consultoría}

La atención de pedidos hacia el data warehouse bajo las modalidades existentes — requerimientos pequeños — implicaba la ejecución de tiempos largos de gestión, 
análisis e implementación para modelos de data warehouse. Por tales razones, se requería la identificación de una nueva forma de atención.

Con la consultoría se relevó la situación actual e, igualmente, se identificó el radio de acción en data warehouse bajo el cual se requería la nueva forma de atención. En un análisis de los tipos de requerimientos se definió lo siguiente:

i. Requerimientos grandes, de más de 1000 horas de ejecución.

ii. Requerimientos medianos, de 500 a 1000 horas de ejecución.

iii. Requerimientos pequeños, menores a 500 horas de ejecución.

iv. Requerimientos FIFO, que son reportes rápidos de modelos productivos del data warehouse. No exceden los cinco días.

Figura 6. Modelo de atención de requerimientos data warehouse

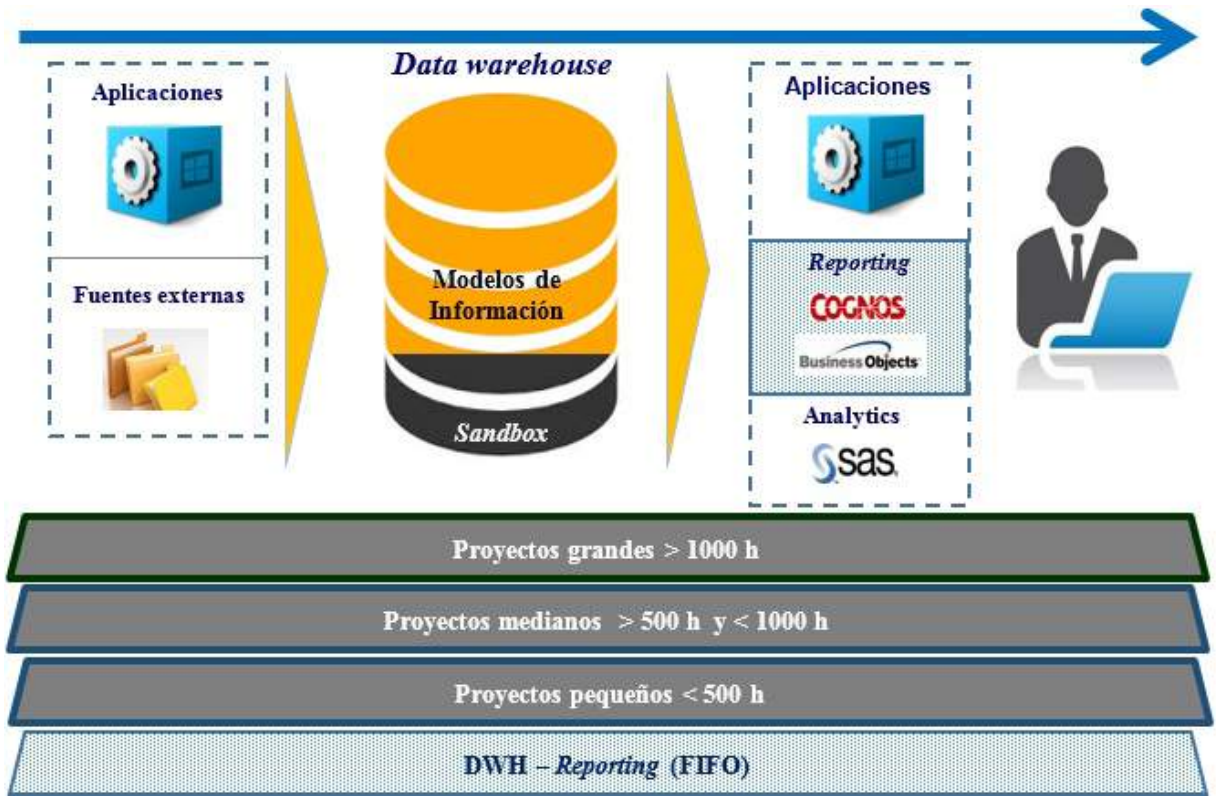

Fuente: BCP

Del mismo modo, el análisis determinó que los requerimientos grandes y medianos tenían estas características:

i. La mayoría de los requerimientos eran regulatorios, y necesitaban una gestión y ejecución detallada por ser de naturaleza compleja. 
ii. Las expectativas de los usuarios se adecuaban a los tiempos requeridos.

iii. Si bien en esfuerzo estos requerimientos eran proporcionalmente más grandes que los pequeños, estos últimos representaban un número mayor. Ejemplo: número de requerimientos atendidos en el primer semestre del 2014: pequeños (67), medianos o grandes (8).

Por otro lado, el análisis determinó que los requerimientos pequeños tenían las siguientes características:

- Menor complejidad.

- Las expectativas de los usuarios era una mejora en el time to market, ya que consideraban que los tiempos eran excesivos para soluciones tácticas pequeñas.

- Algunos usuarios habían comenzado a contratar personal de la Unidad de Sistemas para desarrollar estos pedidos tácticos en sus sandbox sin pasar por el área de sistemas, con lo cual se generaban problemas de duplicidad de información, estabilidad operativa y riesgo operativo.

- Cada vez que los usuarios solicitaban un control de cambios a su requerimiento, de acuerdo a la metodología tradicional, se aceptaba el cambio siempre y cuando no impactara en el negocio (otro requerimiento priorizado), pero la modificación se realizaba al final del pedido inicial.

Asimismo, se validó que los requerimientos FIFO cumplían con un time to market aceptable. Con la información proporcionada se elaboró un proceso de priorización para la aplicación de la metodología Agile data warehouse (ver tabla 4): 
Tabla 4. Matriz de priorización

\begin{tabular}{|c|c|c|c|c|c|c|c|}
\hline & 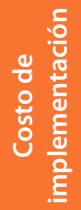 & 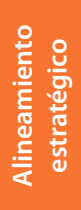 & $\begin{array}{l}\frac{\text { 은 }}{\text { 는 }} \\
\text { 듬 } \\
\text { 응 }\end{array}$ & 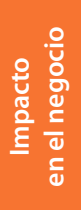 & 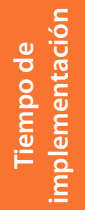 & 厄ँّ & $\begin{array}{l}\text { Orden de } \\
\text { ejecución }\end{array}$ \\
\hline Peso & 2 & 2 & 3 & 3 & 2 & & \\
\hline $\begin{array}{l}\text { Implementar metodología en } \\
\text { requerimientos grandes y medianos }\end{array}$ & 1 & 5 & 4 & 3 & 1 & 35 & 3 \\
\hline $\begin{array}{l}\text { Implementar metodología en } \\
\text { requerimientos pequeños }\end{array}$ & 3 & 5 & 4 & 5 & 3 & 49 & 1 \\
\hline $\begin{array}{l}\text { Implementar metodología en } \\
\text { requerimientos FIFO }\end{array}$ & 5 & 3 & 3 & 2 & 5 & 41 & 2 \\
\hline
\end{tabular}

Nota: Pesos de 1 a 3, donde 1: No contribuye, y 3 contribuye totalmente.

Valores de evaluación de 1 a 5, donde 1: poco importante o muy costoso, y 5 muy importante o poco costoso.

Elaboración propia

De acuerdo con la evaluación realizada, se implementó la metodología en los requerimientos pequeños y en los FIFO. Se tomó una muestra de 15 requerimientos pequeños del primer semestre del 2014 y se identificó que el tiempo promedio de atención era de 83 días.

Figura 7. Proceso de atención de requerimientos en data warehouse

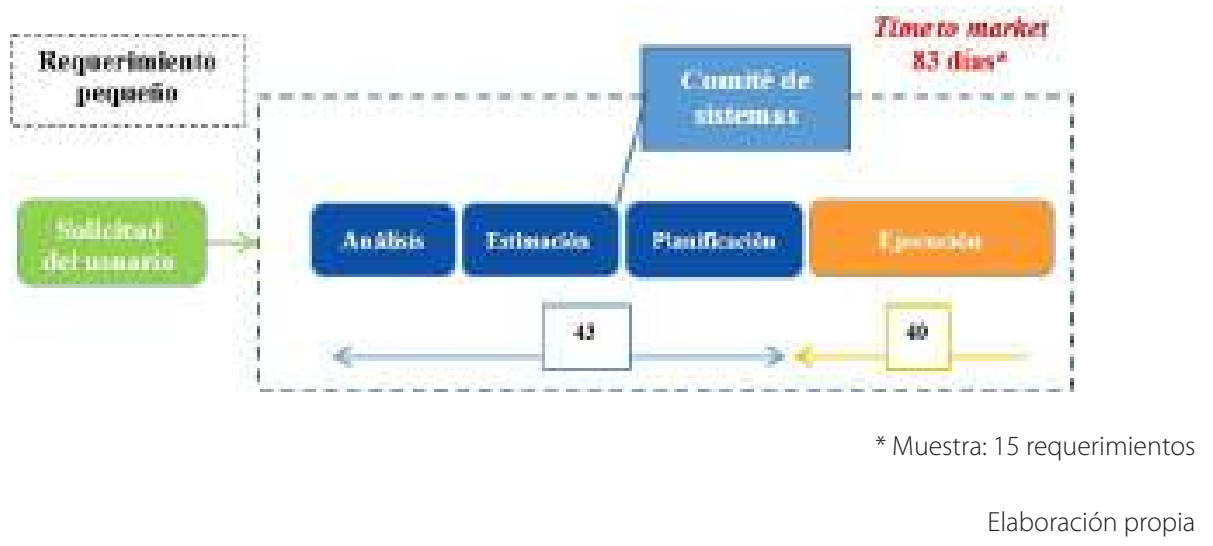

En la figura 7 se aprecian las etapas del proceso de atención de requerimientos: análisis, donde se determina la factibilidad de la solución; estimación del pedido y posterior presentación al Comité de Sistemas para su aprobación; luego el 
requerimiento se planifica en tiempos, costos, entre otros aspectos, y se desarrolla la solución en la etapa de ejecución. Se concluyó que los tiempos eran demasiado extensos, debido principalmente a lo siguiente:

- En la etapa de análisis y estimación no existía dedicación exclusiva de recursos, puesto que estos se asignaban en la etapa de planificación después de que el requerimiento era aprobado por el Comité de Sistemas.

- Este comité se reunía una vez por semana, y si el requerimiento no era aprobado por falta de tiempo debía pasar a la siguiente semana, por lo cual su aprobación podría darse incluso hasta después de un mes.

- En la etapa de ejecución no se permitía la planificación de trabajo en paralelo con los requerimientos (multitasking), ya que al no tener recursos dedicados las tareas se ejecutaban secuencialmente.

- Cualquier cambio que el usuario solicitaba, se evaluaba el impacto en el negocio. Si se aprobaba, este cambio se concretaba una vez terminado el pedido inicial, sin considerar la priorización interna del usuario. Este modelo tenía su fundamento en el retrabajo que tendría que hacer la Unidad de Sistemas al incluir en medio del desarrollo una tarea adicional (Modelo de Mckinsey para el BCP).

\subsection{2 Ámbitos de mejora}

Culminado el análisis, se identificaron dos ámbitos de mejora: la primera en las etapas de gestión (análisis, estimación y planificación) y la segunda en la etapa de ejecución.

Mejoras en la etapa de análisis, estimación y planificación:

- $\quad$ Agilización de las tareas operativas.

- Línea de atención exclusiva con recursos dedicados.

- Tareas paralelas en cada etapa del proceso.

Mejoras en la etapa de ejecución:

- Reducción del time to market de los requerimientos con la implementación de modelos ágiles para requerimientos pequeños, utilizando la metodología Scrum, que brinda mayor valor al negocio en el menor tiempo, con desarrollos por iteraciones (de dos a cuatro semanas) y reuniones a lo largo del proyecto. 
- Participación activa y constante de los usuarios, convirtiéndose en un recurso dentro del desarrollo del proyecto.

- Factibilidad de realizar controles de cambio en los sprint en el proceso de desarrollo, priorizando de acuerdo a su impacto en el negocio.

Por otro lado, se consideraron los siguientes criterios de atención para usar esta metodología:

- Tiempo de ejecución menor a 500 horas.

- Necesidades no regulatorias. Las empresas reguladoras exigen una serie de requisitos en el desarrollo de los requerimientos.

- Requerimientos no multiaplicativos. Debido a que la interacción entre aplicaciones es bastante elevada no se recomienda el uso de procesos metodológicos distintos.

- Generación de reportes de información.

- Cargas de campos nuevos al data warehouse.

- Carga de información a través de data entry.

- Pasar a Producción nuevos campos o modificar lógicas existentes.

- No incluye formalizar modelos existentes de los sandbox.

\subsubsection{Ejecución y resultados del piloto}

Se puso en funcionamiento un piloto con el fin de comprobar las mejoras que se lograrían en términos de tiempo y costos. Para esto, se tomaron como base aproximadamente 15 requerimientos que cumplieran los criterios para ser desarrollados y que también se hayan efectuado en el esquema tradicional. En la ejecución del piloto se cumplieron las etapas de análisis y estimación con recursos dedicados para estas tareas. Luego de la etapa de estimación, no se solicitó la aprobación del Comité de Sistemas; por este motivo, la planificación de los requerimientos fue continua y así se ahorró tiempo de espera de la priorización. Por último, para la etapa de ejecución se tomó como marco de referencia la metodología Scrum, y se dividieron las tareas en sprint. Al final del piloto, se lograron los siguientes beneficios: 
- Reducción del tiempo en $62 \%$ en el time to market total.

- En la etapa de análisis, estimación y planificación se alcanzó un ahorro de tiempo del $81 \%$ con respecto al modelo anterior.

- En la etapa de ejecución se observaron los siguientes beneficios:

- Se ahorró en 43 \% el tiempo, con respecto al modelo anterior.

- Se mejoró la desviación de plazos, con un indicador del $4 \%$ de desviación con relación a un 12 \% en el modelo tradicional.

- Se realizaron en promedio tres Sprint por requerimiento. Esto permitió que se pudieran hacer entregas parciales a los usuarios, paralelizar los procesos y aminorar los tiempos de la entrega final.

- En un $20 \%$ de los requerimientos se realizaron controles de cambio solicitados por el negocio, que con el nuevo modelo no tuvieron que esperar al final del requerimiento para desarrollarlos.

- En un $10 \%$ de los requerimientos el usuario solicitó no culminar con el pedido, pues con los sprint entregados ya satisfacía su necesidad de información para la toma de decisiones; ahorrándose costos adicionales de implementación, y se aseguró el uso de los modelos del data warehouse.

- Como resultado de la reducción del tiempo, se contó con mayor capacidad de atención de requerimientos.

- Se garantizó la calidad de entregable debido a la participación constante y activa del usuario. 
Figura 8. Resultados del piloto

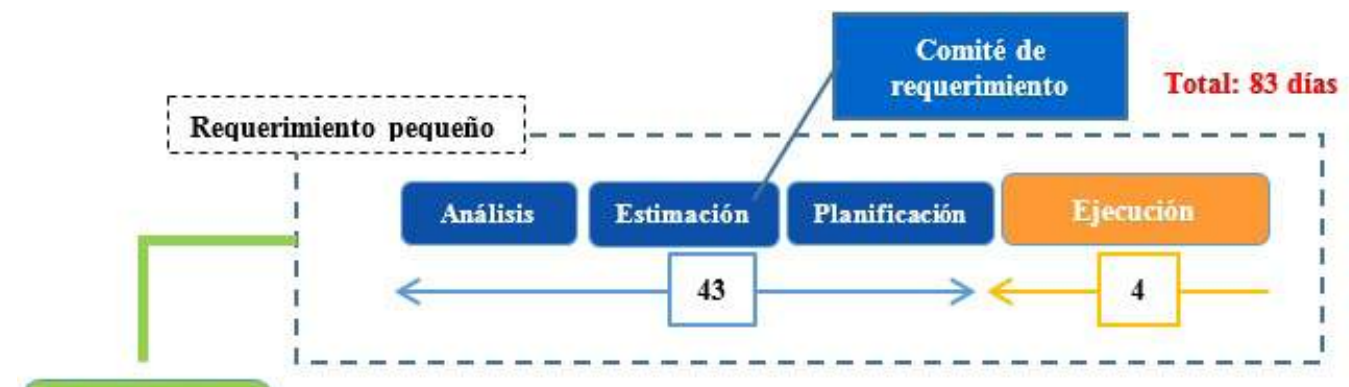

Solicitud

del usuario

Reducción $62 \%$

Total: $\mathbf{3 1}$ días

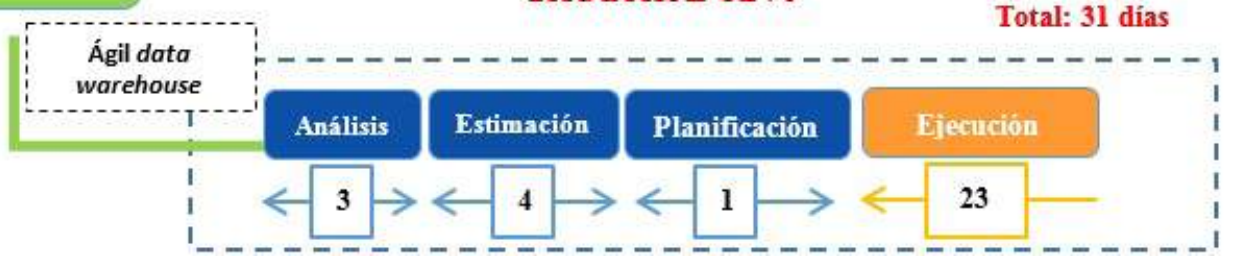

Elaboración propia

\subsubsection{Comité de Gobierno Ágil}

Luego de culminado el piloto, y con el objetivo de velar por el seguimiento del nuevo proceso metodológico, se implementó un Comité de Gobierno Ágil (en adelante comité ágil); conformado por la gerencia del Chief Data Officer (CDO) y las principales gerencias de negocio, así como por la gerencia de Business Intelligence y la gerencia de Project Manager.

Las principales funciones del comité ágil son las siguientes:

- Aprobar y priorizar nuevas iniciativas.

- Controlar el presupuesto asignado.

- Garantizar el cumplimiento de las reglas Agile data warehouse.

- Medir y gestionar la satisfacción de usuario.

La tabla 5 presenta una descripción de los integrantes de este comité y de sus roles: 
Tabla 5. Integrantes del Comité de Gobierno Ágil

\begin{tabular}{|l|l|}
\hline \multicolumn{2}{|c|}{ Integrantes } \\
$\begin{array}{l}\text { Dirección Comité y satisfacción } \\
\text { del usuario }\end{array}$ & $\begin{array}{l}\text { Liderar el comité para que alcance los objetivos definidos en el periodo } \\
\text { establecido. }\end{array}$ \\
\hline Secretario & $\begin{array}{l}\text { Llevar el registro y seguimiento de los pendientes y puntos de acción que } \\
\text { se defina en cada reunión de comité. }\end{array}$ \\
\hline Responsable de priorización & Generar y validar el modelo de priorización y su ejecución. \\
\hline Responsable de reglas ágil & $\begin{array}{l}\text { Validar que las definiciones y reglas establecidas sean ejecutadas } \\
\text { correctamente. }\end{array}$ \\
\hline $\begin{array}{l}\text { Responsable de manejo del } \\
\text { cambio }\end{array}$ & $\begin{array}{l}\text { Apoyar la adopción de la nueva forma de trabajo por parte de los usuarios } \\
\text { yen general de todo el personal comprendido en la iniciativa. }\end{array}$ \\
\hline Responsable de presupuesto & $\begin{array}{l}\text { Monitorear la utilización del presupuesto y sus resultados; y en conjunto } \\
\text { con las áreas usuarias definir la proyección presupuestal. }\end{array}$ \\
\hline $\begin{array}{l}\text { Responsable de satisfacción } \\
\text { usuario }\end{array}$ & $\begin{array}{l}\text { Validar el sentir del usuario en el servicio que se está recibiendo. Si } \\
\text { bien tiene que ver con la calidad del trabajo, también es importante su } \\
\text { percepción sobre los servicios recibidos. }\end{array}$ \\
\hline
\end{tabular}

Elaboración propia

\subsubsection{Formalización del proceso}

Luego de formar el comité ágil, se inició el proceso de formalización con el propósito de desplegar el modelo a las unidades de negocio de la organización. Para esto, se tomó como marco de referencia la metodología Scrum y se adecuó a los procesos del BCP. De manera general, en el proceso participan los siguientes roles:

- Comité ágil, que vela por el cumplimiento del modelo. Se reúne mensualmente para revisar los requerimientos ágiles desarrollados y el seguimiento de indicadores.

- Dueño del programa, que vela de manera detallada por que el modelo se ejecute según lo establecido y elabora y presenta los indicadores de seguimiento al comité ágil. Este rol se le asignó al gerente de Bl y se reúne semanalmente con todo el equipo Scrum para realizar el seguimiento a los indicadores.

- Usuario de negocio, que solicita el requerimiento de información.

- Dueño del producto, cuyo rol corresponde a la oficina del CDO y es la voz del usuario. Cumple con asegurar una comunicación clara sobre el producto y los requisitos con el equipo Scrum. Además, tiene como función elaborar y priorizar los requerimientos ágiles dentro de su gerencia, por el valor que estos generan al negocio. Durante el desarrollo, el dueño del 
producto puede solicitar la modificación del alcance; por esta razón se podría aplicar un control de cambios. De no exceder el $20 \%$ del esfuerzo estimado inicialmente, se atendería de inmediato; en caso contrario, sería agregado al grupo de trabajos que han sido identificados pero que no han sido completados (Backlog).

- Scrum Master, dedicado a monitorear y validar el correcto desarrollo de los requerimientos, gestionar riesgos y resolver los impedimentos tanto externos como internos que afectan al equipo Scrum. Se reúne todos los días con el equipo Scrum y revisa las estimaciones, planificaciones y problemáticas dentro de cada requerimiento.

- El Equipo Scrum, que presenta los entregables finalizados al dueño del producto para su validación previa, y luego el dueño del producto lo valida en conjunto con el usuario solicitante.

Figura 9. Modelo ágil data warehouse

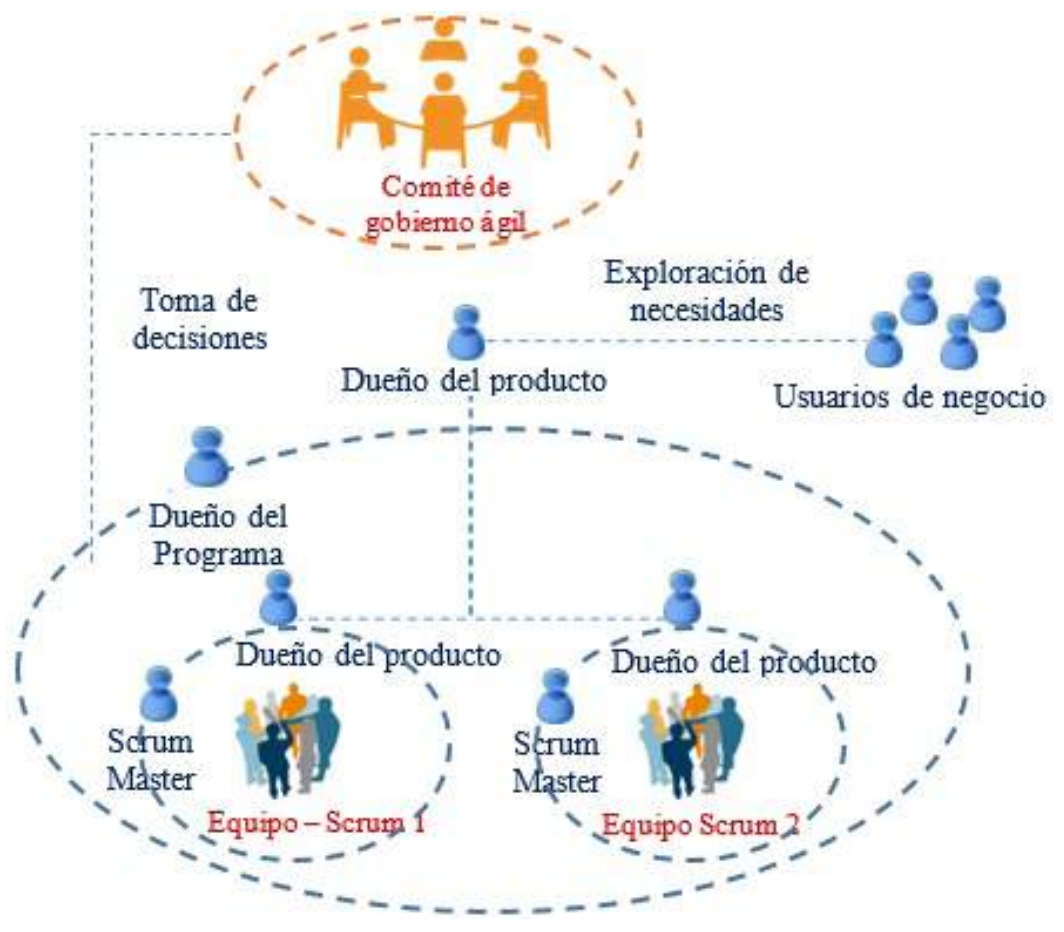

Elaboración propia 


\subsubsection{Implantación del modelo en las áreas de negocio}

Luego de culminada la formalización del proceso metodológico, se implantó el modelo con la aprobación del CIO y el Comité de Sistemas del BCP. Se planteó un proceso general de atención de los requerimientos:

i. El usuario y el dueño del producto exploran la necesidad de información.

ii. El dueño del producto prioriza los requerimientos y solicita a data warehouse el pedido; en caso de que ocurriera algún conflicto de prioridades ellos lo definen. Además, si hubiera la posibilidad de generar entregables parciales se realiza la solicitud.

iii. El equipo de data warehouse analiza el pedido, su factibilidad, y efectúa la estimación y planificación del pedido.

iv. El equipo de data warehouse coordina con el proveedor la ejecución del pedido usando el nuevo modelo de desarrollo de Agile data warehouse.

v. Una vez que la solución se encuentra desarrollada por el proveedor, data warehouse revisa la atención junto con el dueño del producto.

vi. El dueño del producto revisa con el usuario la solución y da el conforme. Cabe resaltar que el usuario participa de manera recurrente en la etapa de ejecución.

Figura 10. Implantación del modelo ágil en áreas de negocio

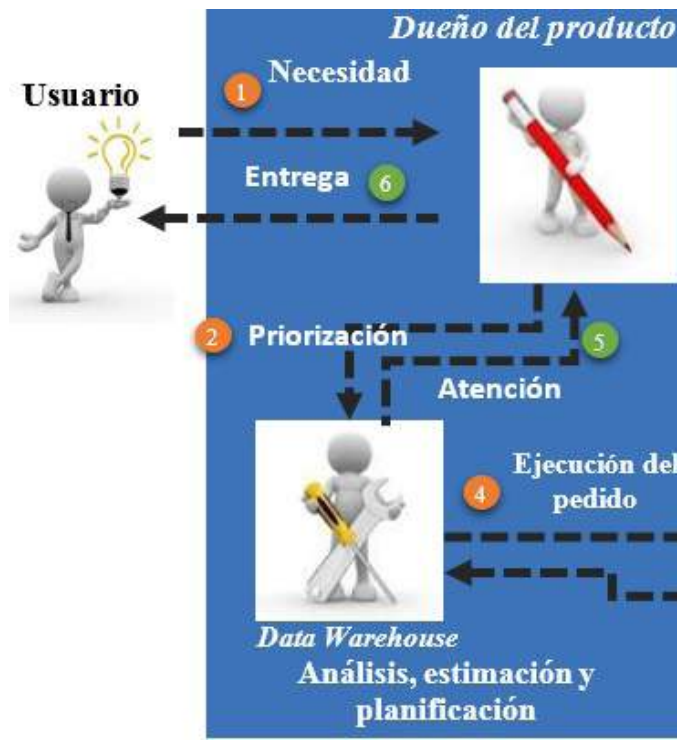

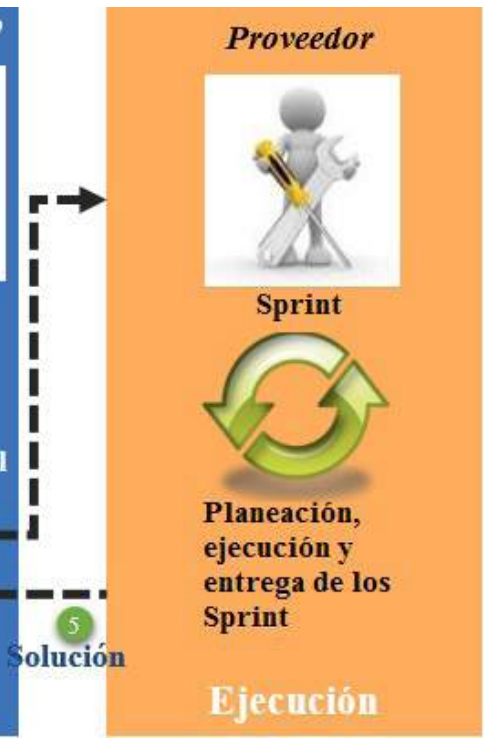

Elaboración propia 
El despliegue de este modelo se inició en enero del 2015. En ese periodo se trabajó con los usuarios los pedidos del primer trimestre del 2015; luego, se planificó el presupuesto y se solicitó su aprobación al Comité de Sistemas. En ese periodo, el comité ágil revisó los indicadores; una vez finalizado el primer trimestre del 2015 se presentó un informe al Comité de Sistemas y se solicitó el presupuesto correspondiente para el siguiente trimestre. Este proceso se ejecuta cada trimestre. En el caso del primer trimestre del 2015, luego del seguimiento, los resultados fueron los siguientes:

- Se ejecutó el $94 \%$ de lo presupuestado para pedidos ágiles de proyectos pequeños (menores a 500 horas).

- Las áreas de negocio que participaron en el despliegue fueron aquellas recurrentes en pedidos hacia data warehouse.

Figura 11. Presupuesto y ejecución primer trimestre del 2015

Distribución por gerencias

\# Ágiles: 65

\# Esfuerzo: 19472

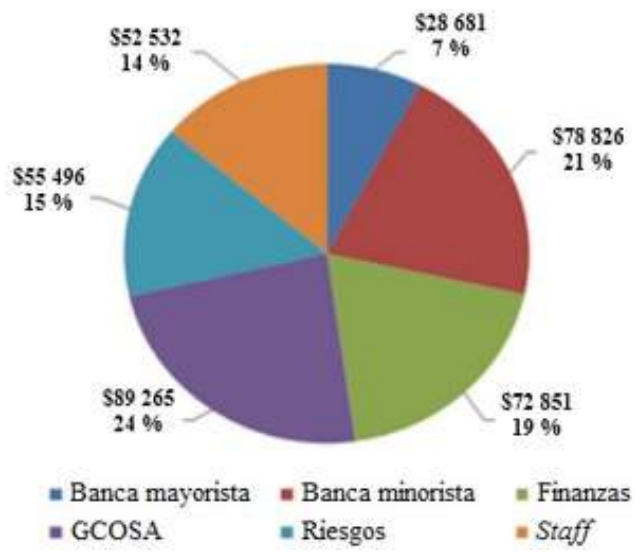

Elaboración propia

\subsection{Seguimiento y control del proyecto}

Durante el proyecto, y luego de finalizar el proceso de implantación del nuevo modelo Agile data warehouse, fue necesario asegurar que el proceso metodológico estuviera en marcha y genere los beneficios esperados. Para esto fue preciso desarrollar las siguientes actividades:

- Verificar si el proyecto cumplió el alcance requerido.

- Verificar que las capacitaciones hayan culminado.

- Verificar que las áreas usuarias estén obteniendo los resultados esperados. 
- Verificar que las áreas usuarias no estén solicitando pedidos a través del Comité de Sistemas, sino que todo pedido ingrese de manera directa al data warehouse.

- Verificar que la infraestructura se encuentre disponible (oficina Agile data warehouse y los equipos de video conferencia).

- Verificar que el modelo de atención agile data warehouse ya se encuentre formalmente normado en Sistemas como un modelo de atención adicional.

- Asimismo, se validó la salud del proyecto en las siguientes perspectivas, el cual se encontró, de manera general, en buen estado.

Tabla 6. Indicadores de salud del proyecto

\begin{tabular}{|c|c|c|c|c|}
\hline Nombre & Descripción & Fórmula & $\begin{array}{l}\text { Meta } \\
\%\end{array}$ & Responsable \\
\hline Presupuesto & $\begin{array}{l}\text { Mide el presupuesto } \\
\text { ejecutado con respecto } \\
\text { a lo planificado }\end{array}$ & $\frac{\text { Presupuesto ejecutado }}{\text { Presupuesto planificado }}$ & 100 & $\begin{array}{l}\text { Gerencia } \\
\text { ejecutiva del } \\
\text { proyecto }\end{array}$ \\
\hline Proveedor & $\begin{array}{l}\text { Mide el avance } \\
\text { del proveedor con } \\
\text { respecto a lo planificado }\end{array}$ & $\frac{\text { Avance ejecutado Proveedor }}{\text { Avance planificado Proveedor }}$ & 90 & $\begin{array}{l}\text { Project } \\
\text { Manager }\end{array}$ \\
\hline $\begin{array}{l}\text { Roadmap } \\
\text { del proyecto }\end{array}$ & $\begin{array}{l}\text { Mide el cumplimiento } \\
\text { de los plazos } \\
\text { establecidos }\end{array}$ & $\frac{\text { Avance ejecutado Proyecto }}{\text { Avance planificado Proyecto }}$ & 100 & $\begin{array}{l}\text { Project } \\
\text { Manager }\end{array}$ \\
\hline Alcance & $\begin{array}{l}\text { Mide el cumplimiento } \\
\text { del alcance esperado }\end{array}$ & $\frac{\text { Alcance logrado }}{\text { Alcance esperado }}$ & 100 & Usuarios \\
\hline Beneficios & $\begin{array}{l}\text { Mide el cumplimiento } \\
\text { de los beneficios } \\
\text { esperados }\end{array}$ & $\frac{\text { Beneficio logrado }}{\text { Beneficio esperado }}$ & 100 & Usuarios \\
\hline
\end{tabular}

En el siguiente gráfico se observan las desviaciones de los indicadores de salud del proyecto, sobre la base de estos rangos: 
Figura 12. Desviación de indicadores de salud del proyecto

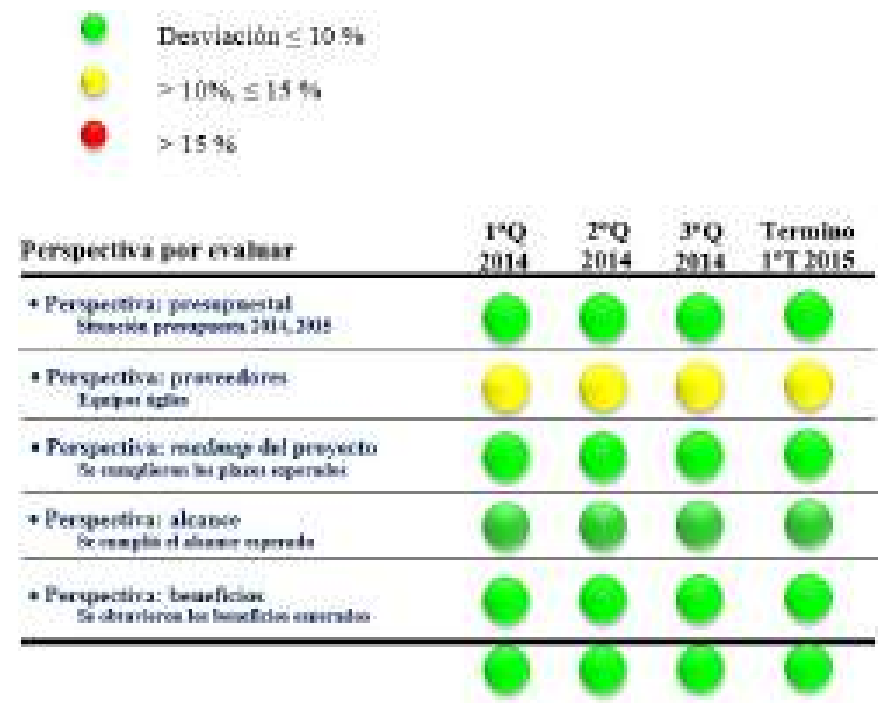

Elaboración propia

\subsection{Cierre}

Después de la puesta en marcha del nuevo proceso metodológico Agile data warehouse, se procedió con la revisión del nuevo modelo para asegurar su correcto funcionamiento. En la tabla 7 se puede apreciar una recopilación de casos de éxito:

\section{Tabla 7. Casos de éxito de los usuarios}

\begin{tabular}{|l|l|l|}
\hline \multicolumn{2}{|c|}{ Área } & \multicolumn{2}{c|}{ Solicitud } \\
\hline Banca mayorista & Factura negociable & $\begin{array}{l}\text { Contar con una base de clientes potenciales actualizada } \\
\text { a quienes ofrecerles el producto de facturas } \\
\text { negociables }\end{array}$ \\
\hline $\begin{array}{l}\text { Banca minorista } \\
\text { (banca pyme) }\end{array}$ & $\begin{array}{l}\text { Cobro centralizado por estudio } \\
\text { de poderes }\end{array}$ & $\begin{array}{l}\text { Incremento en los ingresos por comisión de aperturas } \\
\text { de cuentas corrientes }\end{array}$ \\
\hline $\begin{array}{l}\text { Finanzas } \\
\text { (tesorería) }\end{array}$ & $\begin{array}{l}\text { Elaboración de reportes de } \\
\text { insumos para la generación de } \\
\text { margen semanal }\end{array}$ & $\begin{array}{l}\text { Optimizar el proceso del cálculo del margen semanal, } \\
\text { presentado en el Comité de Gestión }\end{array}$ \\
\hline Banca minorista & $\begin{array}{l}\text { Documentación de reportes } \\
\text { para tarjetas de crédito VISA }\end{array}$ & $\begin{array}{l}\text { Contar con la documentación que sustente el } \\
\text { intercambio de información con VISA y así poder } \\
\text { responder las consultas de la auditoría }\end{array}$ \\
\hline $\begin{array}{l}\text { Banca minorista } \\
\text { (división } \\
\text { comercial) }\end{array}$ & $\begin{array}{l}\text { Elaboración de reportes para } \\
\text { Guerra al Cash }\end{array}$ & $\begin{array}{l}\text { Migrar transacciones de ventanilla hacia otros canales } \\
\text { de menor costo }\end{array}$ \\
\hline Riesgos & $\begin{array}{l}\text { Automatización del proceso de } \\
\text { asociación de tipos de crédito }\end{array}$ & $\begin{array}{l}\text { Eliminar el riesgo operativo en el proceso de cálculo } \\
\text { del score behavior de tarjeta de crédito }\end{array}$ \\
\hline
\end{tabular}




\subsection{Beneficios}

- Reducción del tiempo en el ciclo de desarrollo. En la figura 13 se aprecia la reducción del time to market del primer semestre del 2015, conseguida con el modelo Agile data warehouse frente al modelo tradicional (simulación).

Figura 13. Time to market 2015

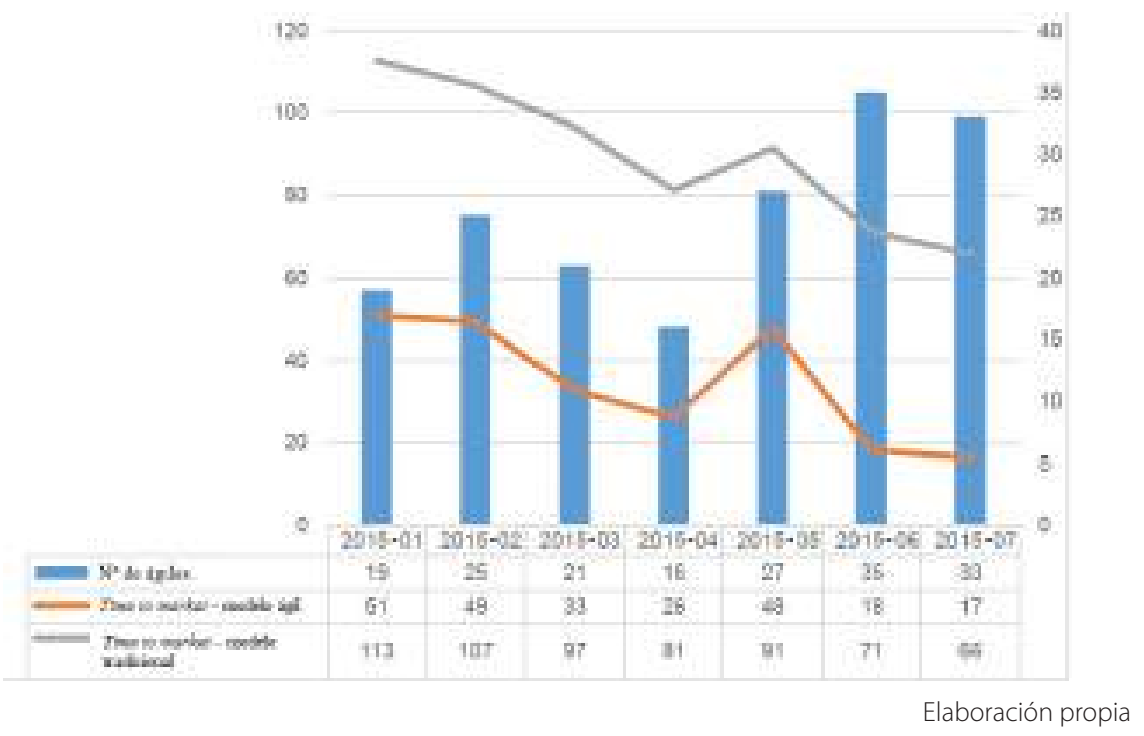

- Reducción de la desviación de plazos por el seguimiento constante y resolución oportuna de los incidentes.

Figura 14. Desviación de plazos de requerimientos 2014 vs. 2015

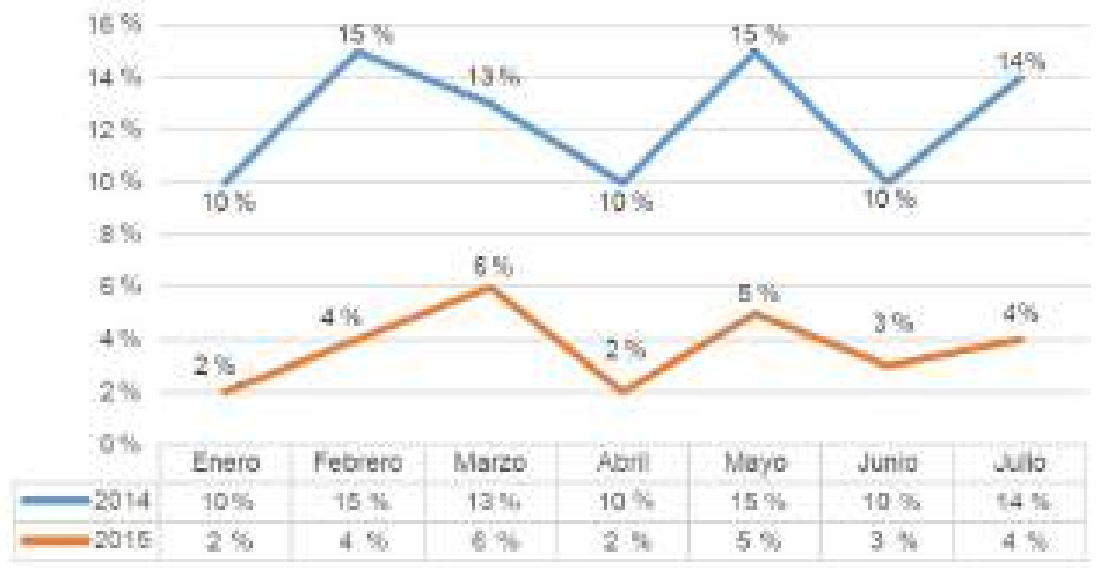

Elaboración propia 
- Mayor atención de requerimientos. Al reducir los tiempos de desarrollo, se generó más oferta de horas para requerimientos que se atendían en los sandbox.

Figura 15. Número de requerimientos atendidos 2014 vs. 2015

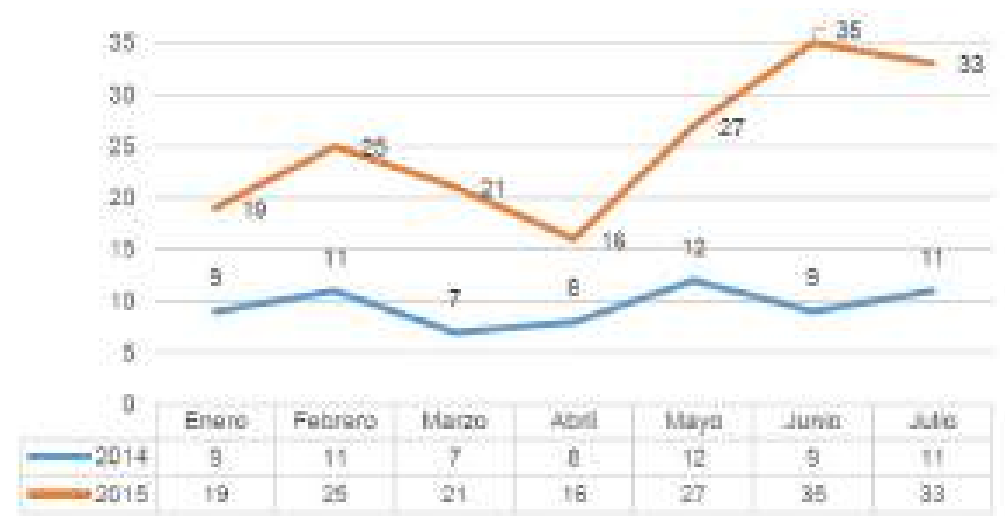

Elaboración propia

- Mejora en eficiencia en la etapa de desarrollo. En la figura 15 se muestra una mayor eficiencia en el desarrollo del requerimiento con respecto al modelo tradicional.

Figura 16. Eficiencia: time to market/esfuerzo 2015

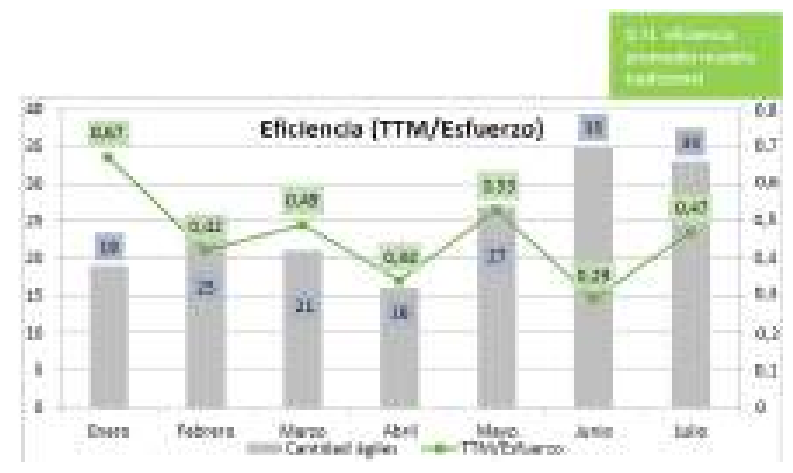

Elaboración propia 


\section{Conclusiones y recomendaciones}

\subsection{Conclusiones}

- Se implementó una nueva metodología para desarrollos ágiles en data warehouse, la cual ha permitido mejorar la flexibilidad y reducir el time to market de los requerimientos. Gracias a ello, las unidades usuarias cuentan con información más oportuna y de mejor calidad, además de lograr una mayor atención de requerimientos.

- El apoyo de la alta gerencia de negocios es un factor clave para que este modelo se institucionalice. La participación trimestral en los Comités de sistemas permite que el modelo siga mostrando los beneficios obtenidos y evaluar las propuestas de mejoras que se deseen implementar.

- Se implementó un Comité de Gobierno Ágil para velar por el seguimiento del nuevo proceso metodológico. Su labor es contribuir con la priorización y atención eficiente de los pedidos de información. Además, permite que los gerentes de negocio que la conforman sigan alentando en sus equipos el uso de esta metodología.

- El presupuesto proyectado posibilitará la utilización del modelo de atención Agile data warehouse para la atención de los requerimientos de las áreas de negocio, lo cual aminorará los tiempos en la priorización de requerimientos.

- La capacitación en metodología ágil es esencial, ya que permite desarrollar el modelo Agile data warehouse de manera eficaz, e identifica a los usuarios en la participación activa de los requerimientos.

- Se ha reducido el riesgo de error y de implementaciones sin valor al negocio.

- La comunicación constante con el usuario directo permite que pueda entender y asimilar los beneficios de este modelo haciéndolo parte de su día a día. Su participación en el modelo es clave para el éxito de la metodología ágil, y con ello se garantiza la calidad del entregable.

- Se cuenta con indicadores que muestran las mejoras realizadas, además del seguimiento al proceso metodológico.

- Se han sentado las bases para explorar los usos de metodologías ágiles en el resto de la organización de sistemas. 


\subsection{Recomendaciones}

- Capacitar continuamente a los usuarios con el fin de que prioricen su participación en los requerimientos, ya que su rol es un punto importante en el éxito del modelo.

- Las unidades usuarias deberán iniciar la migración de los sandbox a los ambientes productivos del data warehouse. De ese modo, se podrá garantizar la estabilidad operativa de sus modelos, así como reducir costos en espacios de almacenamiento y tareas operativas de sus unidades. Además, es importante revisar el trabajo de sistemas que efectúan actualmente en sus áreas, para trasladar esas funciones a la Unidad de Sistemas.

- Iniciar la exploración del uso de metodologías ágiles para el resto de aplicaciones de sistemas.

- Construir un sistema de atención de requerimientos para automatizar el trabajo de ingreso y priorización de los pedidos del Agile data warehouse.

- Evaluar cada seis meses el uso del modelo y plantear las mejoras por implementarse haciendo uso del marco de referencia del modelo Scrum.

- Realizar, a través del comité ágil, un seguimiento al avance de los requerimientos e indicadores.

- Programar, cada seis meses, capacitaciones de refuerzo de uso del nuevo modelo.

\section{Referencias}

Inmon, W. (2002). Building the data warehouse. (3. ${ }^{a}$ ed.). Nueva York, Estados Unidos: John Wiley \& Sons, Inc.

Schwaber, K., y Sutherland, J. (2013). The Definitive Guide to Scrum: The Rules of the Game. Recuperado de http://www.scrumguides.org/docs/scrumguide/v1/scrum-guide-us.pdf

Teradata (2014). Teradata's Agile Development Methodology [página web]. Recuperado de http://www.teradata.at/products-and-services/agile-development-methodology

The Data Warehouse Institute. (2013a). Scrum's role in Agile BI. Recuperado de https://tdwi.org/ articles/2013/07/09/role-of-scrum-in-agile-bi.aspx

The Data Warehouse Institute. (2013b). Ten Mistakes to Avoid in an Agile BI Transformation. Recuperado de https://tdwi.org/research/2013/05/ten-mistakes-to-avoid-in-an-agile-bitransformation.aspx . 